\title{
D meson sensitivity to a system size scan at LHC.
}

\author{
Roland Katz ${ }^{1}$, Jacquelyn Noronha-Hostler ${ }^{2}$, \\ Caio A. G. Prado ${ }^{3}$, and Alexandre A. P. Suaide ${ }^{4}$ \\ 1 SUBATECH, Univ. de Nantes, IMT, IN2P3/CNRS, 44307 Nantes, France \\ 2 Dept. of Physics, Univ. of Illinois at Urbana-Champaign, Urbana, IL 61801, USA \\ 3 Inst. of Particle Phys., Central China Normal Univ., Wuhan, Hubei 430079, China \\ ${ }^{4}$ Inst. de Física, Univ. de São Paulo, C.P. 66318, 05315-970 São Paulo, SP, Brazil
}

\begin{abstract}
Experimental measurements in pA collisions indicate no D meson suppression $\left(R_{\mathrm{pPb}} \sim 1\right)$ but a surprisingly large $v_{2}$. To better understand these results we propose a system size scan at the LHC involving ${ }^{16} \mathrm{OO},{ }^{40} \mathrm{ArAr},{ }^{129} \mathrm{XeXe}$ and ${ }^{208} \mathrm{PbPb}$ collisions. Using Trento+ v-USPhydro+DAB-MOD to make predictions, we find that the $R_{\mathrm{AA}}$ tends towards unity when the system size is decreased, but nonetheless, in the most central collisions $v_{2}\{2\}$ is almost independent of the colliding system. These results are analyzed in light of path length and initial eccentricity variations.
\end{abstract}

1. Introduction. Flow correlations, strangeness enhancement and suppression of hard probes are considered to be three signatures of the Quark-Gluon Plasma. The recent observation of the first two in small hadronic collisions - such as $\mathrm{pp}$ and $\mathrm{pPb}$ - raises many questions on the nature of the created "medium" in these collisions [1/23]. Jet and heavy flavor suppression is not observed in small systems 4, but the CMS collaboration has measured large D meson anisotropies in $\mathrm{pPb}$ collisions [5]. We still dot not understand of how such a significant $v_{2}$ in small systems could be compatible with $R_{\mathrm{AA}} \rightarrow 1[6$. In order to determine the applicability of hydrodynamics in these tiny systems, it was recently proposed to run a system size scan at LHC via ArAr and OO collisions [7, on which various predictions have been made 8 . $\mathrm{D}$ mesons being mostly sensitive to equilibrium dynamics, they appear to be ideal probes of system size effects 9 . Here we investigate these effects on the $R_{\mathrm{AA}}$ and $v_{n}\{2\}$ by varying the colliding nuclei. To do so, we use Trento+v-USPhydro+DAB-MOD [10] with the same soft backgrounds as in [8] and the Langevin set up that gave us the best results in $\mathrm{PbPb}$ collisions 11 .

2. Model Description. The Monte Carlo simulation DAB-MOD 91011, developed to study open heavy favours, is coupled to $2 \mathrm{D}+1$ event-by-event hydrodynamical backgrounds [8]. Heavy quarks are first sampled using FONLL distributions and then propagate with a relativistic Langevin model using the spatial diffusion coefficient from [12. When the heavy quark / medium decoupling temperature $T_{d}$ is reached, the hadronization is finally performed using a hybrid fragmentation/coalescence model. In DAB-MOD the overall magnitude 
of the $R_{\mathrm{AA}}$ is ambiguous as we usually fix the scaling parameter of the Langevin model using $R_{\mathrm{AA}}$ data in most central collisions. Here, we use for all colliding systems the value determined in $\mathrm{PbPb}$ collisions (i.e. $D /(2 \pi T)=2.2$ ).

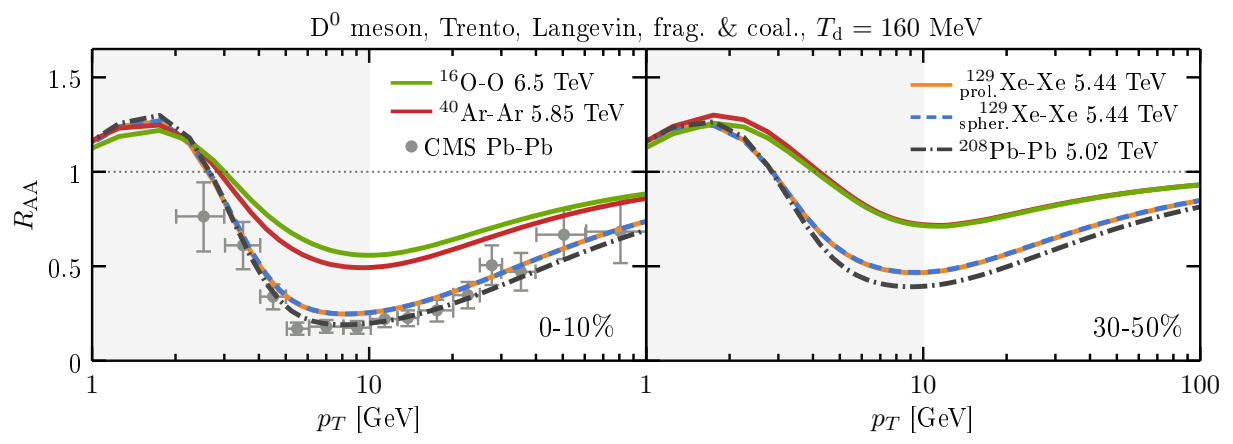

Fig. 1. Direct $\mathrm{D}^{0}$ meson $R_{\mathrm{AA}}$ for $\mathrm{OO}, \mathrm{ArAr}$, XeXe with spherical and prolate initial nuclei, and $\mathrm{PbPb}$ collisions in $0-10 \%$ (left) and $30-50 \%$ (right) centrality classes. Prompt $\mathrm{D}^{0}$ data $(|y|<1)$ from the CMS collaboration for $\mathrm{PbPb}$ collisions [13.

3. Results. In Fig. 1 we first investigate how the system size modifies the $R_{\mathrm{AA}}$ as one moves towards smaller systems. First, central collisions are observed to be more sensitive to system size than mid-central collisions where there is no visible difference between $\mathrm{OO}$ and ArAr even though their system size is different 8 . We expect the $R_{\mathrm{AA}}$ to smoothly approach unity with shrinking system size, as $\left(1-R_{\mathrm{AA}}\right)$ is approximatively proportional to the initial medium radius $\sim A^{1 / 3}$. However, in a future extension to $\mathrm{pPb}$ collisions, we might not reach unity enough to describe the data (like in previous studies with similar frameworks [6]). Finally, the deformation of the Xe nuclei has no influence on the $R_{\mathrm{AA}}$.

For the azimuthal anisotropies $v_{n}$, two factors play a significant role: the size of the system, which can be described by the typical radius of the initial conditions $\mathrm{R}$, and the initial geometrical shape usually characterized by the eccentricities $\varepsilon_{n}[\underline{8}$. Their variations with the colliding system in the two centrality classes considered here are shown in Fig. 2, The systems resulting from OO, $\mathrm{ArAr}, \mathrm{XeXe}$ and $\mathrm{PbPb}$ central collisions have significantly different sizes and eccentricities: the eccentricities increase while the radius decreases. In contrast, in mid-central collisions the eccentricities remain roughly constant when one varies the system size. The mid-central collisions can then be seen as probes of pure system size effects. As measured $\mathrm{D}$ meson data in $\mathrm{pPb}[5]$ correspond to central collisions, they might experience both system size and eccentricities variations compared to large AA collisions. In Fig. 3 (top) we show the $\mathrm{D}$ meson $v_{2}$ in the two different centrality classes. In the $30-50 \%$ mid-central class, the $v_{2}$ of smaller systems are significantly suppressed across all $p_{\mathrm{T}}$. Thus, as $\varepsilon_{2}$ is $\sim$ const. in the mid-central class, the pure effect of the system size plays a dramatic role on the $v_{2}$. In the $0-10 \%$ centrality class, the $v_{2}$ is observed to be roughly independent of the colliding system across all $p_{\mathrm{T}}$. This striking result can be understood by returning to Fig. 2 where we saw that for central collisions the $\varepsilon_{2}$ increases as R 

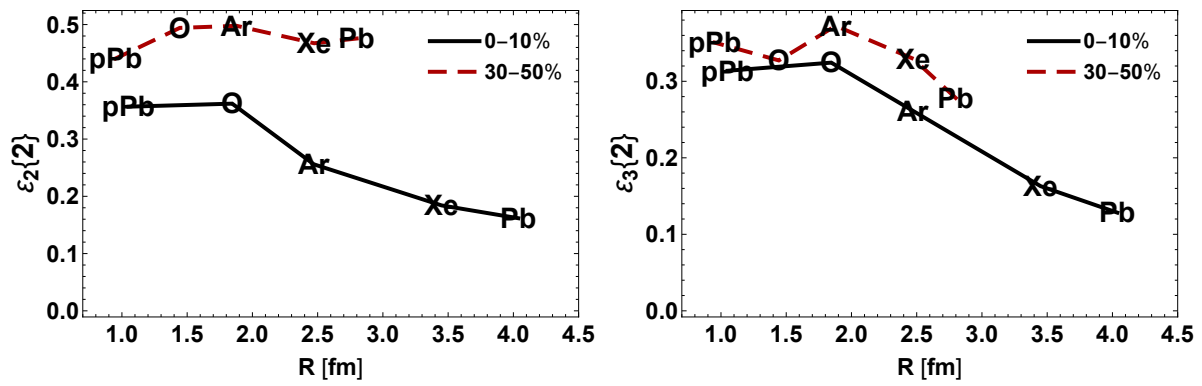

Fig. 2. $\varepsilon_{2}\{2\}$ (left) and $\varepsilon_{3}\{2\}$ (right) versus radius for $\mathrm{OO}, \mathrm{ArAr}, \mathrm{XeXe}$ and $\mathrm{PbPb}$ collisions at the LHC in $0-10 \%$ and $30-50 \%$ centrality classes.

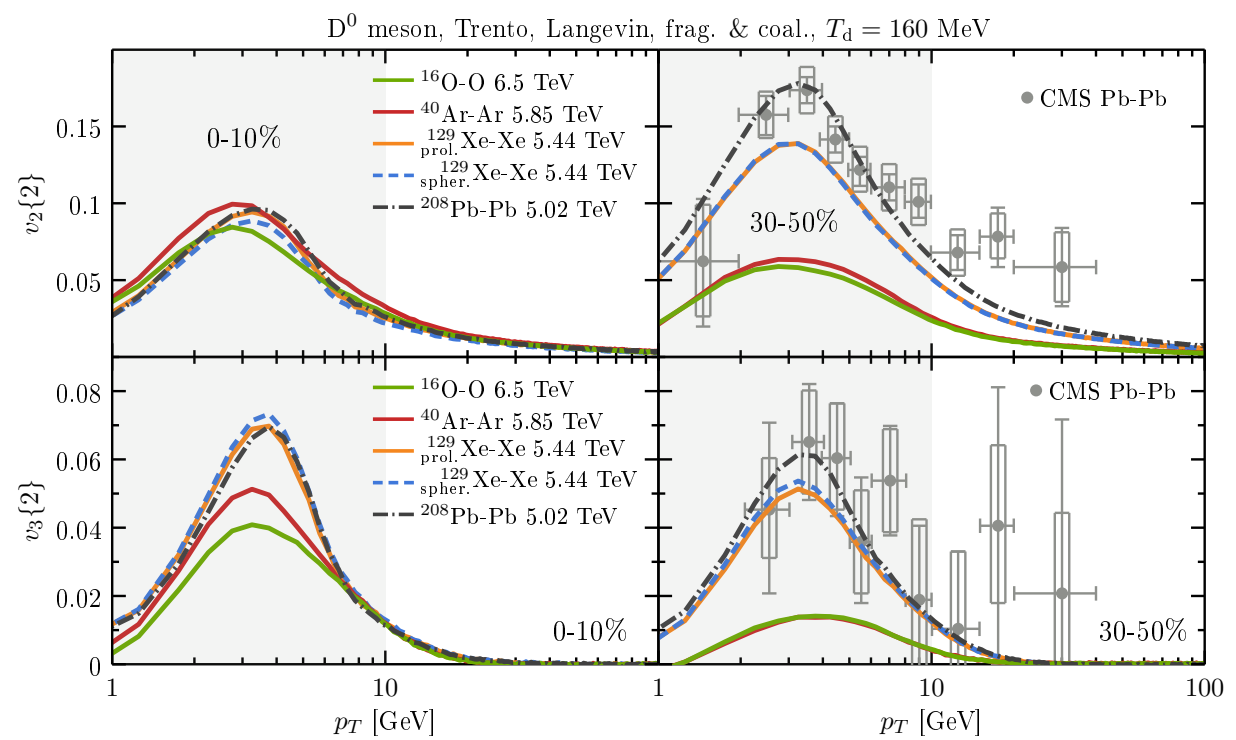

Fig. 3. Direct $\mathrm{D}^{0}$ meson $v_{2}\{2\}$ (top) and $v_{3}\{2\}$ (bottom) for OO, ArAr, XeXe with spherical and prolate initial nuclei, and $\mathrm{PbPb}$ collisions in $0-10 \%$ (left) and $30-50 \%$ (right) centrality classes. Prompt $\mathrm{D}^{0}$ data $(|y|<1)$ from the CMS collaboration for $\mathrm{PbPb}$ collisions 14 .

decreases. Thus, there are now two competing factors that influence the final $v_{2}$ : a suppression effect from decreasing $\mathrm{R}$, like in the mid-central class, and an enhancement effect from increasing $\varepsilon_{2}$. The similarity of the $v_{2}$ curves regardless of colliding system can therefore be explained by the two competing effects roughly compensating each other in central collisions. One can extend these ideas to $\mathrm{pPb}$ collisions: if they have large enough eccentricities (see Fig. 22) $v_{2}$ may not vanish despite the system size shrinking (other effects, e.g. the initial flow, could also contribute). Note finally that in central collisions the $v_{2}$ shows a sensitivity to the deformation of the ${ }^{129} \mathrm{Xe}$ nucleus. In Fig. 3 (bottom), the triangular anisotropies $v_{3}$ are observed to be more sensitive to size effects than 
by eccentricities, i.e. there is a consistent suppression in small systems regardless of the centrality class, even when $\varepsilon_{3}$ changes significantly. Finally, contrasting with the "universality" of $v_{3}\left(p_{\mathrm{T}}\right)$ across centralities usually observed in $\mathrm{PbPb}$ collisions [14, in smaller systems the $v_{3}$ vary strongly with the centrality class.

4. Conclusions. We made predictions for the D meson nuclear modification factors and azimuthal anisotropies for the proposed system size scan at LHC [9]. We find that the $R_{\mathrm{AA}}$ gradually approaches unity as the system size decreases, i.e. as the path lengths shrink. The variations of the $v_{2}$ over the colliding systems depend strongly on two competing factors: the typical system radius $\mathrm{R}$ and the geometry of the initial condition described by its eccentricity $\varepsilon_{2}$. In mid-central collisions, we get a clear hierarchy of the $v_{2}$ between colliding systems, showing the strong influence of the system size itself, as $\varepsilon_{2}$ is nearly constant over the different systems. In central collisions the suppression of $v_{2}$ due to the decreasing $\mathrm{R}$ is counterbalanced by an enhancement coming from an increasing $\varepsilon_{2}$, leading to roughly equivalent $v_{2}\left(p_{\mathrm{T}}\right)$ across the colliding system scan. Although $\varepsilon_{3}$ increases with decreasing $\mathrm{R}$ in central collisions, $v_{3}$ is more sensitive to $\mathrm{R}$ itself and, thus, one observes a suppression following the system size hierarchy regardless of the centrality class. Finally, we find that in small systems $v_{3}$ decreases with centrality, whereas it is known to be almost constant in $\mathrm{PbPb}$ collisions. The latter can now be explained by a balance between a suppression effect from path length reduction and an enhancement from $\varepsilon_{3}$ increase with centrality.

Acknowledgments. The authors thank Fundação de Amparo à Pesquisa do Estado de São Paulo (FAPESP) and Conselho Nacional de Desenvolvimento Científico e Tecnológico (CNPq) for support. R.K. is supported by the Region Pays de la Loire (France) under contract No. 2015-08473. C.A.G.P. is supported by the NSFC under grant No. 11890714 and 1186113100, MOST of China under Project No. 2014CB845404. J.N.H. acknowledges the support of the Alfred P. Sloan Foundation and from the US-DOE Nuclear Science Grant No. de-sc0019175.

\section{References}

1. V. Khachatryan et al. [CMS Collaboration], Phys. Lett. B 765, 193 (2017)

2. J. Adam et al. [ALICE Collaboration], Nature Phys. 13, 535 (2017)

3. C. Aidala et al. [PHENIX Collaboration], Nature Phys. 15, no. 3, 214 (2019)

4. S. Acharya et al. [ALICE Collaboration], arXiv:1906.03425 [nucl-ex]

5. A. M. Sirunyan et al. [CMS Collab.], Phys. Rev. Lett. 121, no. 8, 082301 (2018)

6. Y. Xu et al., Nucl. Part. Phys. Proc. 276-278, 225 (2016)

7. Z. Citron et al., arXiv:1812.06772 [hep-ph]

8. M. D. Sievert and J. Noronha-Hostler, Phys. Rev. C 100, no. 2, 024904 (2019)

9. R. Katz, C. A. G. Prado, J. Noronha-Hostler and A. A. P. Suaide, arXiv:1907.03308

10. C. A. G. Prado et al., Phys. Rev. C 96, no. 6, 064903 (2017)

11. R. Katz, C. A. G. Prado, J. Noronha-Hostler, J. Noronha and A. A. P. Suaide, arXiv:1906.10768 [nucl-th]

12. G. D. Moore and D. Teaney, Phys. Rev. C 71, 064904 (2005)

13. A. M. Sirunyan et al. [CMS Collaboration], Phys. Lett. B 782, 474 (2018)

14. A. M. Sirunyan et al. [CMS Collab.], Phys. Rev. Lett. 120, no. 20, 202301 (2018) 